\title{
La construction de l'admiration dans le roman Béatrix
}

\author{
Alexandra Delcamp
}

La Comédie humaine ${ }^{1}$ affectionne les personnages supérieurement grands, supérieurement beaux ou supérieurement bons. Certes, ils ne sont pas les plus nombreux et ne sont pas même systématiquement les protagonistes, ${ }^{2}$ mais ces personnages d'exception contrastent avec l'abondante médiocrité humaine : on ne peut s'empêcher de les admirer. ${ }^{3}$ Bien entendu, ce contraste seul ne saurait suffire à expliquer notre admiration, laquelle dépend, dans le roman, d'une construction complexe. Avant de nous pencher sur le fonctionnement très spécifique de l'œuvre romanesque, il est utile de définir ce qu'est l'admiration, précisément et indépendamment du roman.

La définition cartésienne permet de considérer l'admiration comme un processus dont on peut distinguer deux étapes essentielles : d'abord, elle est « subite surprise de l'âme ${ }^{4}$; c'est-à-dire qu'elle est, en premier lieu, une émotion spontanée qui n'implique aucune construction ni réflexion préalable. Ensuite, elle porte «à considérer avec attention les objets qui lui semblent rares et extraordinaires $»^{5}$, c'est-à-dire qu'elle est, en second lieu, une opération de l'esprit qui mobilise notre volonté de connaitre. En résumé, l'admiration

1 Cette œuvre monumentale rassemble plus de go ouvrages, que Balzac a divisé en trois grands ensembles (Études de mœurs, Études philosophiques et Études analytiques), dont le premier est lui-même subdivisé en plusieurs catégories (Scènes de la vie privée, Scènes de la vie de province, Scènes de la vie parisienne, Scènes de la vie politique, Scènes de la vie militaire, Scènes de la vie de campagne). L'objectif de l'écrivain, qui n'avait pas d'abord songé au cycle romanesque, fut plus tard de proposer une «histoire naturelle de la société ». Des ouvrages écrits antérieurement à ce projet y ont été intégrés progressivement. Balzac y a régulièrement apporté des corrections pour assurer l'unité de son œuvre.

2 Le système des personnages reparaissants permet de les faire passer au second plan. Par exemple, dans Le Père Goriot, l'intertextualité permet d'identifier Claire de Beauséant comme un personnage d'exception : on la reconnait pour être l'héroïne de La Femme abandonnée.

3 La définition la plus commune de l'admiration implique nécessairement un objet supérieurement grand et/ou supérieurement beau ou bon. Nous nous basons ici sur la définition du CNRTL, qui fait de l'admiration un «sentiment complexe d'étonnement, le plus souvent mêlé de plaisir exalté et d'approbation devant ce qui est estimé supérieurement beau, bon ou grand. »

4 Cf. René Descartes, Les passions de l'âme, Paris, 1996, art. 70, p. 142.

5 Ibid.

(C) ALEXANDRA DELCAMP, 2022 | DOI:10.30965/9783846766644_008

This is an open access chapter distributed under the terms of the CC BY-NC-ND 4te license De $_{\text {LCamp }}-9783846766644$ 
nous fait passer de la surprise à la curiosité, de l'émotion à l'opération de l'esprit, du hasard ${ }^{6}$ à la volonté.

D'un point de vue phénoménologique, on pourrait traduire ce double mouvement de la manière suivante : d'abord, l'objet d'admiration fait saillie sur ce fond d'indifférence que constitue pour nous notre horizon perceptif, attirant ainsi notre attention. ${ }^{7}$ Puis, l'émotion se transforme en opération de l'esprit vis-à-vis de l'objet d'admiration qui dès lors devient objet de connaissance. Comme dans le monde réel tel que décrit par la phénoménologie, l'admiration pourrait, dans La Comédie humaine, se décomposer en deux étapes : d'abord la rencontre avec l'objet d'admiration, puis la transformation de ce dernier en objet de connaissance. Or si l'on retrouve, chez Balzac, les grandes lignes de ce processus, le statut très particulier de l'œuvre romanesque complique ce découpage et exige certains réajustements.

Ainsi, dans la première étape, la rencontre avec l'objet d'admiration implique dans le roman une construction qu'elle n'implique absolument pas dans le monde réel, car dans l'œuvre de fiction, la rencontre avec l'objet d'admiration dépend non pas du hasard mais bien de la volonté organisatrice et créatrice de l'écrivain. Partant, même son caractère « surprenant » est à nuancer. Le romancier peut certes décider de faire apparaitre subitement l'objet d'admiration dans le récit de manière à nous surprendre, mais là encore, nous devons notre surprise à une construction, ou plus précisément à un «effet de surprise ${ }^{8}$ aménagé par l'auteur. Dans tous les cas, l'admiration implique une construction qui ne débute pas avec la rencontre de l'objet admiré, mais commence parfois bien avant elle. On distinguera donc une étape supplémentaire qui précède les deux autres et au sein de laquelle l'auteur construit cet environnement borné par l'horizon perceptif du roman et où se mettront en place, souvent à l'insu du personnage, parfois à l'insu du lecteur, les conditions propices à la rencontre avec l'objet d'admiration.

6 Hasard qui nous met en présence de l'objet d'admiration, abstraction faite de l'éventualité d'une providence ou de la capacité de l'être humain à se déterminer lui-même.

7 Dans son article "Phénoménologie de la surprise : horizon, projection et événement», Françoise Dastur revient sur la notion husserlienne d' «horizon », qu'Edmund Husserl avait lui-même empruntée à Leibniz. Développant ses arguments en faveur d'une phénoménologie de la surprise, elle explique que, du point de vue phénoménologique, «ce qui vient à notre rencontre se détache toujours sur un arrière-fond obscur qui constitue comme une réserve ouverte à des explorations futures». Cf. Françoise Dastur, «Phénoménologie de la surprise : horizon, projection et événement », in Alter, 2016, $\mathrm{n}^{\circ} 24$. Pour consulter cet article en ligne, voir l'URL suivante : http://journals.openedition.org/alter/412 (consulté le 15 juin 2020).

8 Cf. Raphaël Baroni, La tension narrative. Suspense, curiosité, surprise, Paris, 2007, p. 296-307. 
Létape de la transformation de l'objet d'admiration en objet de connaissance, quant à elle, revêt une importance toute particulière dans La Comédie humaine, car derrière la représentation romanesque de l'objet d'admiration s'esquisse la continuité du monde perceptible en une face «apprésentée ${ }^{9}$ qui constitue, plus que l'objet d'admiration lui-même, le véritable but de la connaissance - aussi bien pour le personnage non-initié que pour le lecteur.

Rappelons que dans le monde réel tel que décrit par la phénoménologie, nous ne nous trouvons jamais en rapport direct avec un objet, mais en rapport indirect, via cet objet, avec « un ensemble de relations de significations dans lequel nous nous trouvons immergés, lesquelles de leur côté renvoient à cette dimension d'ouverture que nous nommons $\left\langle\right.$ le monde $>»^{10}$. Autrement dit, lorsque notre objet d'admiration devient pour nous objet de connaissance, notre volonté de connaitre, en se portant sur l'objet admiré, se porte en réalité sur l'ensemble des relations qu'implique ce dernier. Par conséquent, il s'agit moins de connaitre l'objet d'admiration pour lui-même que de connaitre le monde tel que reconfiguré, sous de nouveaux rapports, par l'apparition de cet objet.

Sur ce point, les choses ne fonctionnent pas si différemment dans le roman balzacien, à ceci près que le processus de transformation de l'objet d'admiration en objet de connaissance obéit, une fois de plus, à une construction romanesque : l'écrivain utilise volontiers l'objet d'admiration pour créer un point de passage vers la connaissance de la face apprésentée du monde.

Il est important de noter dès à présent les difficultés que soulève une telle construction : comme, dans La Comédie humaine, cette face apprésentée s'étend plus ou moins loin dans le champ du connaissable, elle n'est pas également représentable. Sous son aspect le plus simple, elle ne dissimule rien d'autre que le jeu des apparences et des intérêts qui régissent la société. C'est, par exemple, la face dissimulée des choses qu'Henriette de Mortsauf dévoile à Félix dans sa lettre avant que celui-ci ne s'installe à Paris ${ }^{11}$ et plus généralement, celle que tentent de décrypter tous les arrivistes de La Comédie humaine, avec plus ou moins de succès. Dans ce cas, aucun problème particulier ne se pose pour représenter ce qui est dissimulé : tout est potentiellement connaissable et donc potentiellement communicable.

Mais il en va tout autrement dès lors que la face apprésentée du monde atteint les limites du connaissable, voire les dépasse. En effet, comment transmettre

9 Du verbe allemand appräsentieren, utilisé par Husserl dans Cartesianische Meditationen, La Haye, 1973, p. 139.

10 Dastur, «Phénoménologie de la surprise», § 2.

11 Cf. Honoré de Balzac, Le Lys dans la vallée, Paris, 1855. 
l'indicible intuition de l'artiste, du philosophe, du scientifique, du mystique ? Cette configuration n'est pourtant pas rare dans La Comédie humaine. C'est Louis Lambert ${ }^{12}$ et le peintre Frenhofer ${ }^{13}$ quittant le monde sans avoir pu communiquer l'intégralité de leur œuvre au commun des mortels, c'est Antoinette de Langeais ${ }^{14}$ et Félicité des Touches ${ }^{15}$ se retirant au couvent pour prolonger leur amour terrestre en Dieu, c'est encore Séraphîta ${ }^{16}$ rejoignant les cieux. Comment, lorsque le personnage-objet d'admiration est lui-même un génie dépassé par sa propre vision, un artiste abandonnant son art pour s'abîmer en la mort ou encore un amant laissant derrière lui son amour pour s'abîmer en Dieu, pourrait-il nous initier à une face du monde qu'il connait sans pouvoir se la représenter clairement à lui-même ? Comment pourrait-il nous faire connaitre l'irreprésentable ou, en d'autres termes, le monde non pas construit, non pas imité, mais pensé, intuité par Balzac, par-delà les bornes de l'horizon perceptif?

Quelle que soit l'étendue de la face apprésentée du monde, son intelligibilité dépend essentiellement de la construction du personnage-objet d'admiration, ce carrefour central de la connaissance. Pour que sa transformation en objet de connaissance réussisse (dans la mesure où elle le peut), il faut que le personnage-objet d'admiration soit à la fois en capacité de connaitre et de donner à connaitre. Cette construction devient particulièrement complexe quand les objets d'admiration se multiplient dans le roman, et encore davantage lorsque le sentiment amoureux se mêle à l'admiration. Justement, c'est ce qui se produit dans Béatrix. Balzac y met en scène un triangle d'admiration amoureuse. L'histoire de cette admiration amoureuse en quelques mots : Calyste, «l'occasion du drame »17, admire Félicité des Touches, il en tombe amoureux ; Félicité suscite involontairement l'admiration de Calyste pour Béatrix. Calyste aime Béatrix ; dès lors, Félicité suscite volontairement l'admiration de Béatrix pour Calyste ; Béatrix aime peut-être Calyste ; enfin, Félicité admire le cœur pur et la beauté de Calyste. Elle l'aime, elle se refuse à lui. Calyste est au centre du drame, mais c'est un autre personnage qui relie, par un acte de la volonté, tous ces objets d'admiration entre eux, quoique Balzac nous le présente comme «un personnage secondaire». Il s'agit de Félicité des Touches,

\footnotetext{
12 Cf. Honoré de Balzac, Louis Lambert, Paris, 1855.

13 Cf. Honoré de Balzac, Le Chef-d'ouvre inconnu, Paris, 1993.

14 Cf. Honoré de Balzac, La duchesse de Langeais, Lausanne, 1968.

15 Cf. Honoré de Balzac, Béatrix, préf. de Julien Gracq, Paris, 1953.

16 Cf. Honoré de Balzac, Séraphîta, Paris, 1855.

17 C'est ainsi qu'Alain définit Calyste dans «Avec Balzac» (1935), in: Alain, Balzac, Paris, 1999, p. 13-118, p. 9o.
} 
ou plutôt Camille Maupin de son nom de plume, une romancière de génie aux étonnantes capacités de démiurge.

Pour analyser la construction littéraire de l'admiration, le roman Béatrix offre un double intérêt : en même temps qu'il obéit à une construction romanesque visant à susciter l'admiration, il donne à lire la construction romanesque de l'admiration. Nous nous proposons maintenant de suivre une à une les étapes de la construction de l'admiration dans le roman Béatrix, de l'élaboration d'un environnement délimité par un horizon perceptif jusqu'à la transformation de l'objet d'admiration en objet de connaissance, en passant par la construction de l'objet d'admiration lui-même. Par la même occasion, nous tenterons de déterminer le rôle que joue le sentiment amoureux dans le processus de l'admiration et éventuellement dans sa construction.

\section{La construction de l'environnement}

La première étape de l'admiration correspond, chez Balzac, à la construction de l'environnement de la diégèse. Dans La Comédie humaine, celle-ci s'opère assez souvent dès le début du roman et occupe parfois plusieurs dizaines de pages, retardant d'autant l'apparition des personnages et le commencement de l'action. Béatrix n'échappe pas à cette règle : les trente premières pages sont consacrées à la Bretagne, puis à la ville de Guérande, puis à l'hôtel des Guénic. Pour Balzac, ce procédé est stratégique : «Sans la topographie et la description de la ville, sans la peinture minutieuse de cet hôtel, les surprenantes figures de cette famille eussent été peut-être moins comprises. Aussi les cadres devaient-ils passer avant les portraits. ${ }^{18}$

Loin de ne servir qu'à créer un «effet de réel $»^{19}$ qui cumulerait les «petits faits vrais ${ }^{20}$, la description balzacienne reflète le monde de la diégèse et permet d'établir des correspondances entre les personnages et leur environnement. Placée au début du roman, elle laisse présager des couleurs du drame à venir, des tempéraments et parfois même des physionomies des personnages, de la même façon que la pension du Père Goriot annonçait, avec force équivalences, la repoussante madame Vauquer. À la manière d'un ciel d'orage, les lieux, les objets, l'architecture se chargent de toujours plus de sens; le sens ainsi cumulé est retenu ou plutôt contenu jusqu’à ce que tel ou tel autre élément du

18 Balzac, Béatrix, p. 44.

19 Cf. Barthes Roland, «L'effet de réel », in Communications, 1968, $\mathrm{n}^{\circ}$ 11, p. 84-89.

20 Le terme apparait dans la lettre de Stendhal à Balzac datée du 30 octobre 1840. 
monde qui se déploie dans la diégèse le fasse éclater. Dès lors, tout, entre les bornes de l'horizon perceptif, est susceptible d'annoncer l'objet d'admiration.

Cet horizon perceptif, Balzac le délimite dès l'incipit de Béatrix : « La France, et la Bretagne particulièrement, possède encore aujourd'hui quelques villes complètement en dehors du mouvement social qui donne au dix-neuvième siècle sa physionomie. $»^{21}$ Le monde dans lequel nous nous apprêtons à entrer sera, semble-t-il, en tout point étranger au monde familier des contemporains de Balzac. C'est un monde qui ignore le «mouvement social» de son siècle. Un monde devenu rare, qui n'existe plus qu'à travers «quelques villes». Un monde vestige, en quelque sorte. Cette opposition entre monde ancien et monde moderne, entre immobilisme et mouvement est encore renforcée par les connaissances intertextuelles du lecteur. Ne retrouve-t-on pas dans cet incipit une fidèle image de la province et de son « caractère de simplicité ${ }^{22}$, telle que Balzac la décrivait dans le préambule d'Eugénie Grandet ? Est-il possible, pour le lecteur assidu de La Comédie humaine, de concevoir la province de Balzac autrement que par opposition à Paris, ce lieu de dispersion où, comme le décrit le narrateur dans les premières pages de La Fille aux yeux d'or, «tout fume, tout brûle, tout brille, tout bouillonne, tout flambe, s'évapore, séteint, se rallume, étincelle, pétille et se consume ${ }^{23}$ ? Spontanément, le lecteur associe cette opposition entre la France et ces «quelques villes» de Bretagne à une opposition entre Paris et la province.

Cette intuition sera confirmée lorsque la description se resserrera sur l'hôtel des Guénic : «Dans cette situation, cette famille, qui n'est plus rien pour personne en France, serait un sujet de moquerie à Paris : elle est toute la Bretagne à Guérande. ${ }^{24}$ La moquerie parisienne, cette forme de dispersion, s'oppose à la faculté centralisatrice de la famille du Guénic. ${ }^{25}$ Paradoxalement, c'est

\footnotetext{
21 Balzac, Béatrix, p. 29.

22 Balzac, Eugénie Grandet, Paris, 1855, p. 12.

23 Balzac, La Fille aux yeux d'or, Lausanne, 1968, p. 7.

24 Balzac, Béatrix, p. 37.

25 La question de la centralité chez Balzac a été évoquée à de nombreuses reprises, notamment dans les travaux de Jean-Dominique Goffette qui fait de l'île de la Cité le lieu symbolique d'une centralité mythique, antérieure à la Révolution, par opposition aux boulevards parisiens, lieu de « la France révolutionnée et la grande confusion égalitaire » (Jean-Dominique Goffette, « D’un imaginaire à l'autre : boulevards balzaciens, boulevards flaubertiens », in Romantisme, 2006, $\mathrm{n}^{\circ}$ 134, p. 34). On retrouve également cette idée chez Chantal Massol qui voit dans la société secrète balzacienne l'occasion d'un «principe de centralité secret» (Chantal Massol, Une poétique de l'énigme. Le Récit herméneutique balzacien, Genève, 2006, p. 299), faisant contrepoids à l'éclatement de la centralité provoqué par la Révolution. Il y aurait donc, chez Balzac, un idéal de « concentration des énergies » (Ernst Robert Curtius, Balzac, trad. Michel Beretti, Paris, 1933, p. 101-124) s'opposant à la
} 
au moment où l'horizon du lecteur semble le plus restreint qu'il entrevoit la possibilité d'un horizon perceptif plus étendu que ce à quoi il s'était d'abord attendu : selon toute probabilité, le récit se déroulera à Guérande, mais Guérande ne sera pas tout l'horizon du récit. Paris commence à se profiler dans cet horizon.

L'horizon perceptif délimité, il reste encore beaucoup à décrypter. Nous ne savons toujours pas quel sens donner à toutes ces informations historiques, ces détails topographiques et architecturaux qui jalonnent la description. Pourquoi, par exemple, le narrateur ne cesse-t-il d'évoquer les marais salants ? Y a-t-il réellement un sens dissimulé derrière cet élément du paysage ou s'agit-il, exceptionnellement, d'un «petit fait vrai »?

Plus généralement, ce qui nous est décrit ne nous permet pas d'en prévoir les implications dans la suite du récit. Nous nous trouvons face à un lieu épargné par la linéarité du temps et dont toutes les époques semblent converger vers une sorte de centre sacré où les origines mythiques et immémoriales des Celtes alterneraient indifféremment avec les origines non moins mythiques de la chevalerie et du catholicisme. Une beauté pure, primitive et dont l'adjectif qui la qualifie le plus souvent est «nä̈ve ${ }^{26}$. Une force inébranlable, «stationnaire ${ }^{27}$ qui, à l'image des maisons, châteaux et murailles guérandaises, « résiste $[\ldots]$ à tout $»^{28}$.

Si rien de tout cela ne suffit à nous rendre la suite du récit prévisible, mais que, malgré tout, nous ne pouvons nous empêcher de formuler des hypothèses, c'est que nous sommes sous le coup d'un « effet de curiosité $»^{29}$ construit par Balzac. Lécrivain, tout en nous dissimulant ce qu'il n'est pas encore tant que sachions, nous révèle l'essentiel : l'environnement breton est supérieurement grand et supérieurement beau. En d'autres termes, le «cadre » du roman Béatrix est déjà, en lui-même, digne d'admiration.

Il en ressort que pour construire l'environnement que délimite l'horizon perceptif de la diégèse, la stratégie balzacienne ne mise pas sur la construction

dispersion postrévolutionnaire des énergies individuelles, opposition que l'on retrouve parfois, dans les mœurs, entre Paris et la province. Plus généralement, cette faculté centralisatrice a été remarquée par Georges Poulet, cette fois sur le plan de l'œuvre-monde : «La Comédie humaine est une sphère dont chaque partie se met en rapport avec toutes les autres et avec l'ensemble par un mouvement qui sétend jusqu'à la circonférence, comme l'ensemble se met en rapport avec chaque partie par une pression d'autant plus grande que cette partie est proche de l'endroit où toutes les pressions convergent, c'est-à-dire le centre. » (Georges Poulet, Les Métamorphoses du cercle, Paris, 2016, p. 319.)

26 Balzac, Béatrix, p. 29-44.

27 Ibid., p. 32.

28 Ibid., p. 31.

29 Baroni, La tension narrative, p. 21. 
d'un monde qui constituerait pour nous un « fond d'indifférence », c'est-à-dire d'un monde si familier qu'il en deviendrait, à l'image du monde réel, indifférent, dépourvu de sens a priori. Au contraire, en faisant de l'environnement un objet d'admiration en soi, l'écrivain nous prépare à rencontrer de «surprenantes figures » et chez elles, l'admirable. Il y a en quelque sorte pré-construction de l'objet d'admiration, objet qui selon toute probabilité ne fera pas saillie par contraste avec son environnement, mais au contraire en constituera le prolongement. Cette admiration originelle obéit certes à sa propre construction : usant cette fois d'une esthétique du contraste basée sur l'évocation d'un comparé quasi-absent du texte (Paris et la vie moderne), Balzac nous donne progressivement à voir l'exceptionnalité de l'environnement breton. Ainsi, l'écrivain nous pousse à prévoir que, paradoxalement, l'exceptionnalité ne constituera pas l'exception mais la règle de notre horizon perceptif.

\section{L’introduction de l'objet d'admiration dans le récit}

Il s'agit maintenant d'observer le traitement réservé, dans le récit, aux trois principaux objets d'admiration, à savoir Calyste, Félicité et Béatrix. Ces trois personnages y sont introduits à différentes vitesses, mais tous trois ont pour point commun d'en être longtemps absents. Balzac les intègre dans le récit l'un après l'autre, mais aussi l'un en fonction de l'autre.

Le premier dont il est question, c'est Calyste. ${ }^{30}$ Mais il en est question in absentia. Avant qu'il n'apparaisse dans le récit, il faut passer par tous les Guénic, ainsi que par les personnages qui leur sont proches. Ces derniers font l'objet d'une telle multiplication de prosopographies et de portraits moraux, et constituent l'occasion de si nombreuses pauses descriptives, que le récit semble ne pas pouvoir commencer. Durant tout ce temps, Calyste n'apparait jamais qu'en filigrane et toujours relativement aux personnages qui lui sont liés. Une lecture attentive permet néanmoins de se rendre compte qu'il s'impose comme un centre vers où tout le petit univers des du Guénic converge : «[d]ans cet hôtel [...] [i]l n'y avait d'autre intérêt mêlé à ceux-là [le roi et la religion catholique] que l'attachement de tous pour le fils unique, pour Calyste, l'héritier, le seul espoir du grand nom des du Guénic. » ${ }^{31}$ Mademoiselle du Guénic ne fait d'exception à sa rigueur économe que pour « ce qui concern[e] Calyste, l'enfant

\footnotetext{
30 Le prénom de ce personnage annonce déjà son exceptionnalité : Calyste vient du superla-

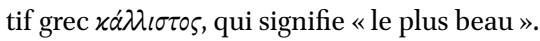

31

Balzac, Béatrix, p. 5 o.
} 
adoré de la maison $»^{32}$. Si Gasselin, le serviteur, « se consid[ère] comme faisant partie de la famille $»^{33}$, c'est parce qu'«il a[...] joué avec Calyste ${ }^{34}$. Mademoiselle de Pen-Hoël, dont le nom est également en voie d'extinction a, «dès la naissance de Calyste, formé le projet de transmettre ses biens au chevalier en le mariant à l'une des nièces que devait lui donner la vicomtesse de Kergarouët-Pen-Hoël » ${ }^{35}$. De plus, Calyste porte le nom du saint qui « dev[ait] toujours protéger les Guénic ${ }^{36}$. Tout, dans le monde de la diégèse, tend vers ce grand absent.

Or, avant que le récit ne nous soumette ce portrait manquant, un nouveau personnage surgit, a priori étranger à l'environnement des Guénic, mais profondément lié à Calyste : il s'agit de Félicité des Touches. Calyste, dit-on, en est amoureux. Elle aussi nous apparait in absentia, par personnages interposés. Mais il y a une différence : tandis que Calyste apparait sans qu'on parle de lui, Félicité n'apparait que parce qu'on parle d'elle. Ainsi, son portrait est pris en charge non pas par le narrateur omniscient, mais par un relai de personnages qui, bien souvent, rapportent eux-mêmes des paroles entendues ailleurs. Jusqu'à ce que le narrateur nous dévoile son portrait «objectif », c'est-à-dire en focalisation zéro, Félicité nous est présentée sous un angle pour le moins inattendu chez une femme que l'on sait adorée de Calyste : elle ne brille que par sa «monstruosité ».

Contrairement à Calyste, ce centre vers qui tout converge, Félicité est rejetée dans une périphérie lointaine, celle du «singulier», du monstrueux, bref, de l'ex-centricité au sens le plus littéral : «- [Grand-Jacques] m’a raconté les plus singulières choses sur notre voisine, mademoiselle des Touches, mais si singulières que je ne veux pas les croire. Calyste ne serait pas si assidu chez elle, il a bien assez de bon sens pour s'apercevoir de pareilles monstruosités. ${ }^{37} \mathrm{Ce}$ mot de «monstruosité » réapparaitra à de nombreuses reprises dans la bouche des Guérandais. C'est encore l'abbé Grimont qui développe cet aspect de Félicité avec le plus de virulence : chez cette « gaupe », cette « gourgandine», cette « femme de mœurs équivoques », tout n'est que monstruosité, c'est-à-dire altérité maximale. Elle «mange [...] sa fortune avec la société du diable» (comédiens, musiciens, écrivains, etc.), ne fréquente pas les églises, n’est même pas

\begin{tabular}{ll}
\hline 32 & Ibid., p. 59. \\
33 & Ibid., p. 57. \\
34 & Ibid. \\
35 & Ibid., p. 63-64. \\
36 & Ibid., p. 45. \\
37 & Ibid., p. 75.
\end{tabular}


royaliste, vit dans « un paradis de Mahomet ». ${ }^{38}$ Elle se situe donc à l'extrême opposé de Dieu, à l'extrême opposé de la foi, à l'extrême opposé des valeurs anciennes, à l'extrême opposé de la Bretagne, et par conséquent, à l'extrême opposé du microcosme guérandais.

Pour le moment, ce n'est donc pas en tant qu'objet d'admiration que Camille Maupin se démarque de l'horizon perceptif. Au contraire, elle inspire la crainte et le dégoût, sentiments qui, a priori et contrairement à l'admiration, dissuadent du désir de connaitre plutôt qu'ils ne l'encouragent. Pourtant, cette exceptionnalité, certes monstrueuse, est le signe par lequel Balzac nous annonce l'un des personnages-objets d'admiration les plus importants du roman. Il faut bien sûr garder à l'esprit que ce premier portrait est pris en charge par des narrateurs-témoins dont tout l'horizon perceptif s'étend, au plus large, à Guérande.

Il est également à noter que, jusquà présent, l'absence prolongée de l'objet d'admiration est le moyen privilégié par lequel Balzac prépare le lecteur à rencontrer l'objet d'admiration. C'est paradoxalement par cette absence que l'écrivain attire notre attention sur cet objet et nous le rend visible ; et c'est par cette absence qu'il l'introduit dans notre horizon perceptif et l'inscrit dans un «à-venir» du récit. Cependant, il n'utilise pas tout à fait les mêmes procédés en fonction de l'objet d'admiration concerné. Ainsi, Calyste s'esquisse progressivement dans notre horizon perceptif, tandis que Camille Maupin y surgit sans que son nom ait été précédemment évoqué. Le premier nous apparait lentement et aiguise toujours davantage notre curiosité, la seconde nous prend par surprise. Il est vrai que Calyste nous a été longuement annoncé par l'environnement breton dont il partage, via le portrait de ses parents qui en forment pour ainsi dire la synthèse, les caractéristiques essentielles (origines nobles immémoriales, force, beauté et naïveté), tandis que Camille nous a été à peine suggérée par cette possible altérité que représentent Paris et la vie moderne. Mais dans les deux cas, ces objets d'admiration sont introduits par l'intermédiaire des personnages qui composent leur environnement proche (pour Calyste) ou lointain (pour Camille).

Quant à Béatrix, seul le titre permet pour l'instant d'envisager son introduction dans la diégèse : rien, dans le récit, ne la laisse présager. Et nous ne sommes pas au bout de notre attente : tant que nous n'aurons pas pleinement reconnu Calyste et Camille pour nos objets d'admiration, Béatrix restera absente du récit. Nous verrons que son apparition dépend entièrement de sa construction en tant qu'objet d'admiration.

$38 \quad$ Ibid., p. 78. 


\section{La construction de l'objet d'admiration}

Lorsqu'on a enfin affaire à Calyste in praesentia et que son portrait en focalisation zéro nous est donné à lire, nous retrouvons concentrées en lui toutes les qualités que nous lui avons prévues. C'est le moment où nous le rencontrons en tant qu'objet d'admiration. Mais l'apparition de ce « chef-d'œuvre humain » ${ }^{39}$ est aussi, et très rapidement, l'occasion d'ouvrir une nouvelle perspective sur Félicité, a priori diamétralement opposée à ses précédents portraits, dès lors ravalés au rang de commérages. Calyste se fait narrateur-témoin, et prend en charge ce nouveau portrait de Félicité, qu'il expose à sa mère : « Ne répétez pas les calomnies qui courent sur elle : Camille est artiste, elle a du génie et mène une de ces existences exceptionnelles que l'on ne saurait juger comme les existences ordinaires. $»^{40}$ Pour la première fois, Félicité nous est présentée sous le jour du «supérieurement grand ». Les critères « ordinaires », semble-t-il, ne permettent pas de la juger objectivement. Mais on se méfie : la parole d'un jeune homme amoureux vaut-elle davantage que les commérages de personnages bornés?

Enfin, un narrateur-régisseur nous annonce que Félicité va nous être présentée depuis un point de vue omniscient :

Il devient maintenant nécessaire d'expliquer les rumeurs qui planaient sur le personnage que Calyste allait voir. [...] Expliquer par quel enchaînement de circonstances s'est accomplie l'incarnation masculine d'une jeune fille, comment Félicité des Touches s'est faite homme et auteur; pourquoi, plus heureuse que madame de Staël, elle est restée libre et se trouve ainsi plus excusable de sa célébrité, ne sera-ce pas satisfaire beaucoup de curiosités et justifier l'une de ces monstruosités qui sélèvent dans l'humanité comme des monuments, et dont la gloire est favorisée par la rareté ? car, en vingt siècles, à peine compte-t-on vingt grandes femmes. Aussi, quoiqu'elle ne soit ici qu'un personnage secondaire, comme elle eut une grande influence sur Calyste et qu'elle joue un rôle dans l'histoire littéraire de notre époque, personne ne regrettera de sêttre arrêté devant cette figure un peu plus de temps que ne le veut la poétique moderne. ${ }^{41}$

Étonnamment, le point de vue objectif tend à confirmer à la fois le point de vue subjectif de Calyste et celui des Guérandais. Félicité fait partie des rares «grandes femmes » et par là, elle se situe du côté du supérieurement grand. Mais elle n'en reste pas moins une «monstruosité ». D'ailleurs, Balzac ne nous dit pas qu'il compte la réhabiliter dans sa supériorité pour nous détromper de

\footnotetext{
39 Ibid., p. 84.

$40 \quad$ Ibid., p. 89 .

41 Ibid., p. 91-92.
} 
ce qu'auraient pu nous faire croire les commérages ; au contraire il projette de «justifier» sa monstruosité. Ni la perspective de Calyste, ni celle des Guérandais n'est erronée : elles décrivent bien une seule et même réalité.

S'en suit un panorama complet de Félicité (alliant biographie détaillée, prosopographie et portrait moral) dont chaque élément souligne l'exceptionnalité de cette « femme de génie » et suscite l'admiration du lecteur. Tout, chez Félicité, est prodigieux : «son cœur pur $»^{42}$, son érudition, sa mémoire, son goût pour les arts et les sciences, sa richesse, sa célébrité, sa beauté. Compte tenu de la longueur du portrait, peu d'espace est somme toute consacré à son autre facette ; mais cet espace suffit amplement à nous «justifier » sa monstruosité : «malgré ces promesses irritantes et assez cachées aux profanes, le calme de cette physionomie a je ne sais quoi de provoquant. Elle effraie par son silence et par ce regard profond d'une profonde fixité. [...] Chacun tremble de rencontrer les corruptions étranges d'une âme diabolique. [...] On a peur de trouver en elle je ne sais quoi de vierge, d'indompté. La femme forte ne doit être qu'un symbole, elle effraie à voir en réalité. $»^{43}$

La monstruosité de Félicité n'est autre que le prolongement naturel de son insoutenable supériorité. Chez elle, c'est l'indicible mystère, traduit à deux reprises par l'expression «je ne sais quoi », qui fait trembler, comme on tremblerait devant une divinité - substantif qui d'ailleurs la qualifiera trois fois dans le roman. En effet, elle en a les caractéristiques : si elle terrifie, c'est parce qu'elle fascine au-delà du supportable. Et c'est là que nous, lecteurs, rencontrons véritablement cet objet d'admiration, car nous associons dès lors Félicité à une exceptionnalité qui touche au divin ; c'est-à-dire, dans le monde balzacien, au Spécialisme. ${ }^{44}$ Là encore, c'est l'intertextualité qui permet de comprendre si tôt que l'objet d'admiration « Camille » dépasse de loin tous les autres. Félicité, qui avait été introduite dans le récit par la périphérie la plus lointaine, puis présentée par le narrateur comme un "personnage secondaire », commence à s'esquisser comme un possible « centre» de l'admiration.

Bien que nous pensions avoir d'ores et déjà pris toute la mesure de l'exceptionnalité de Camille, Balzac, tout au long du roman, parvient à nous démontrer que nous sommes encore loin du compte. Cette femme, qui contemple le monde depuis des «hauteurs désespérantes ${ }^{45}$, qui est capable de le décrypter,

\footnotetext{
42 Ibid., p. 94.

43 Balzac, Béatrix, p. 102-103.

44 Dans Louis Lambert, Balzac distingue « trois degrés de l'homme » : «Instinctif, [l'Homme] est au-dessous de la mesure ; Abstractif, il est au niveau ; Spécialiste, il est au-dessus. Le Spécialisme ouvre à l'homme sa véritable carrière, l'infini commence à poindre en lui, là il entrevoit sa destinée. » (Balzac, Louis Lambert, p. 215.)

Balzac, Béatrix, p. 114.
} 
de le lire avec une acuité terrifiante, nous est elle-même difficile à lire. Ses actes, ses comportements commencent le plus souvent par nous être « inexplicables ». Ils sont comme autant de signes, d'indices enroulés ${ }^{46}$ sur eux-mêmes qui nécessitent d'être déroulés par le récit pour nous devenir lisibles.

Ainsi, à titre d'exemple, nous ne comprenons la raison des refus de Camille à l'égard de Calyste qu'à mesure qu'elle nous est dévoilée : d'abord, la nature des sentiments de la romancière pour le jeune homme est baignée d'une aura de mystère. Le narrateur lui-même parle « d'inexplicables refus ${ }^{47}$; puis il les attribue, «peut-être $»^{48}$, à la « terrible analyse de Camille Maupin ${ }^{49}$ qui a très vite compris que l'adoration que lui voue Calyste est plus de l'ordre du « besoin d'aimer que de l'amour $»^{50}$. Puis, c'est elle-même qui tentera d'expliquer son refus au perplexe jeune homme : «La nature a changé pour moi ses lois en m’accordant encore cinq à six ans de jeunesse. Je vous ai repoussé par égoïsme. Tôt ou tard l'âge nous aurait séparés. $\gg^{51}$ Mais on sent que ce n'est pas là toute la vérité. Enfin, c'est son amant, l'écrivain Claude Vignon, ${ }^{52}$ qui propose une explication si clairvoyante, que Félicité elle-même en est étonnée : « Vous êtes arrivée à Paris éperdument amoureuse de Calyste, disait-il à Félicité; mais vous étiez épouvantée des suites d'une semblable passion à votre âge : elle vous menait dans un abîme, dans un enfer, au suicide peut-être ! Lamour ne subsiste qu'en se croyant éternel, et vous aperceviez à quelques pas dans votre vie une séparation horrible : le dégoût et la vieillesse terminant bientôt un poème sublime. $»^{53}$

Ce procédé qui consiste à « dérouler» la complexe et sublime Félicité tout au long du récit permet de placer le lecteur dans une position quasi-constante d'infériorité vis-à-vis de son objet d'admiration : pour « lire » Félicité, il faut être à sa hauteur ou du moins, comme ce grand esprit Claude Vignon, s'en approcher de très près ; ce que Balzac ne nous permet jamais. En dosant savamment

46 Nous empruntons la métaphore à Gilles Deleuze, qui la développe dans Proust et les signes, mais ici, nous considérons le signe à l'intérieur de la diégèse et tel qu'il peut être « déroulé », via le récit, par une coopération stratégique auteur/lecteur et non pas tel qu'il se présente à l'écrivain indépendamment de l'écriture (Gilles Deleuze, Proust et les signes, Paris, 2014, p. 118-124).

47 Balzac, Béatrix, p. 115 .

48 Ibid.

49 Ibid.

$50 \quad$ Ibid.

51 Ibid., p. 120.

52 Alain qualifie Vignon de «Tirésias» (Alain, «Avec Balzac», p. 90), soulignant ainsi son admirable capacité à décrypter le monde et à en prévoir les développements. 
ce qu'il révèle et ce qu'il dissimule, ${ }^{54}$ l'écrivain créé une distance infranchissable et toujours renouvelée entre le lecteur et son objet d'admiration. Il suscite chez lui la sensation d'un manque d'acuité qui a pour conséquence de placer Félicité toujours plus haut, de la rendre toujours plus admirable, et toujours plus inaccessible. A l'inverse, même si nous admirons l'extraordinaire beauté de Calyste, Balzac nous fait nous sentir d'un esprit supérieur au sien : nous sommes constamment éclairés sur sa naïveté par Camille Maupin.

Enfin, il était temps, Béatrix fait son apparition dans le roman. Elle aussi y apparait in absentia, au prétexte d'une lettre dont elle est l'auteur. C'est Félicité qui, pour une raison absolument obscure, la transmet à Calyste. Il n'y aura pas d'autre introduction au personnage de Béatrix. Avant de la lui laisser lire, la romancière se lance dans un long portrait de la marquise de Rochefide. Originellement, ce portrait n'a pas d'autre but que lui-même. Camille ne cherche ni à montrer Béatrix sous un jour terrible (comme les Guérandais l'avaient fait avec elle), ni même sous un jour admirable. Par sa peinture, elle cherche à atteindre non pas le vraisemblable, mais le vrai. Comme Balzac. ${ }^{55}$

Et en effet, c'est bien d'un exercice de composition romanesque qu'il s'agit. D'ailleurs, on retrouve les éléments qui structurent les portraits des Guénic, décrits plus tôt en focalisation zéro : plongée dans la généalogie, interprétation des traits physiques et moraux, analepses, prolepses, pauses descriptives au sein même de la description (portraits de personnages tiers), intégration de scènes du passé sous forme de dialogue, correspondance entre le contexte historique et la personnalité, etc. Et comme dans les pauses descriptives balzaciennes, l'impression que, à la manière d'un miroir concentrique, ${ }^{56}$ tout l'univers de Béatrix (le roman pour Balzac, le personnage pour Camille) est contenu, quoiqu'encore imparfaitement lisible puisqu'enroulé sur lui-même, noué, attendant d'être dénoué, déroulé par le récit - ou, pour les personnages, par le réel diégétique.

Camille Maupin, cette artiste « totale », dispose même de certains avantages sur Balzac : elle peut, si elle le souhaite, faire entendre la musique, et montrer

\footnotetext{
54 Massol développe cette question dans Une poétique de l'énigme, le récit herméneutique balzacien.

55 Cf. Dominique Massonnaud, Faire vrai. Balzac et l'invention de l'œeuvre-monde, Genève, 2014.

$5^{6} \quad$ L'idée d'observer le monde depuis un miroir concentrique, Balzac l'emprunte à Leibniz, lequel explique dans sa Monadologie que chacune de nos perceptions s'étend à l'ensemble de l'univers et que chaque objet contemplé («substance») représente tout l'univers à sa manière et selon un certain angle de vue (Gottfried Wilhelm Leibniz, La Monadologie. CEuvres philosophiques de Leibniz, texte établi par Paul Janet, Paris, 19oo, t. 1, art. 16, p. 709 et art. 6o, p. 716).
} 
la peinture, ce qu'elle fera pour illustrer son portrait en pointant du doigt une copie du tableau de Miéris. Calyste voit directement l'image, tandis que le lecteur est contraint de se la représenter par le biais d'une description en focalisation zéro. Cette différence n'est pas anodine : elle présage d'un décalage de perception entre Calyste et le lecteur vis-à-vis de l'objet d'admiration Béatrix.

D'une manière générale, cette mise en abîme du romancier qui compose la romancière qui compose un personnage permet une double construction de l'admiration qui elle-même permet un dédoublement perceptif. Ainsi, en nous montrant Béatrix avec plus de distance que ne la voit Calyste, Balzac modère notre admiration pour elle tout en accentuant celle du jeune homme. De même, en nous donnant à voir Camille en train d'exercer brillamment ses talents de romancière, Balzac renforce notre admiration pour elle.

La construction de l'admiration se multiplie encore et obéit à une double coopération stratégique auteur/lecteur ${ }^{57}$ : la nôtre, avec Balzac ; celle de Calyste, avec Camille. Or, en ce qui concerne la coopération stratégique entre la romancière et le jeune homme, quelque chose ne fonctionne pas; les choses ne se produisent pas comme l'avait prévu la romancière : « L'admiration peinte sur le visage du jeune homme était plus excitée par la peinture que par le peintre dont le faire manquait son but. $»^{58} \mathrm{Il}$ faut dire que Calyste, doté d'une trop « vive imagination » et de trop peu d'esprit, n'est pas un lecteur modèle. Toute la partie peu flatteuse du portrait lui échappe. Il semble même que, du moins pendant un temps, il n'en retienne que l'aspect sensuel, ou visuel, plus précisément.

Or ce n'est pas un hasard si le portrait de Béatrix tracé par Camille Maupin, dont l'art de romancière consiste entre autres à susciter des images, est marqué par la picturalité (ekphrasis, ${ }^{59}$ hypotyposes, ${ }^{60}$ champ lexical de la peinture, ${ }^{61}$ etc.). C'est cette représentation visuelle, produit du croisement entre la description romanesque et l'imagination de Calyste, qui surgit devant le jeune homme à chaque fois que Béatrix est évoquée : lors de l'ouverture de la lettre, lors de l'interprétation par Camille et Vignon de son contenu (auquel Calyste n'a pas compris grand-chose) et enfin, lorsque Calyste guette l'arrivée de Béatrix sur le port du Croisic. Est-ce si étonnant de la part d'un personnage mieux disposé aux choses des sens qu'à celles de l'esprit ? En effet, Calyste a ceci en

57 Pour les notions de coopération stratégique, de lecteur et d'auteur modèles, cf. Eco Umberto, Lector in fabula, trad. Muriem Bouzaher, Paris, 1985, p. 61-105.

$5^{8}$ Balzac, Béatrix, p. 127.

59 Il s'agit du moment où Camille montre le tableau à Calyste.

6o Balzac, Béatrix, p. 126 : «avec le plus joli pied du monde, avec cette abondante chevelure d'ange que le pinceau de Girodet a tant cultivée et qui ressemble à des flots de lumière. ».

61 Ibid., p. 127 : «lignes», « dessin », « couleurs », «profil», etc. 
commun avec son père que la «pensée » est pour lui un «effort», et a «son siège plus au cœur que dans la tête». Il entretient une sorte de rapport instinctif au monde : il voit, il sent, mais ne perçoit $^{62}$ pas. Dès lors, une question se pose : comment Béatrix, en tant qu'objet d'admiration, pourrait-elle devenir objet de connaissance pour Calyste?

\section{La transformation de l'objet d'admiration en objet de connaissance}

Observons maintenant le jeune breton presser le pas dans le «dédale des marais salants $»^{63}$, porté par l'espoir d'apercevoir Béatrix sur le port du Croisic. Pourquoi ? Mais parce que « [q]uel est le jeune homme plein d'amour débordant et de vie contenue qui n'aurait eu l'idée victorieuse d'aller au Croisic voir débarquer madame de Rochefide, afin de pouvoir l'examiner incognito ? ${ }^{64}$ On n'obtiendra pas davantage que cette généralité assénée par le narrateur omniscient. Il est vrai que, jusqu'à présent, Calyste lui-même se préoccupe fort peu de comprendre les raisons de ses actes ou de ses sentiments. Sa curiosité n'est pas intellectuelle, elle est instinctive.

Son regard se pose sur une caisse. Elle est au nom de «Madame la marquise de Rochefide ». À ce moment précis, ce signe, dans lequel Béatrix est tout entière contenue, fait véritablement saillie sur l'horizon perceptif d'un Calyste en extase, au sens heideggérien du terme. ${ }^{65}$ Là se trouve pour Calyste l'occasion d'une véritable «ekstase temporelle "66 (ekstatikon), car le jeune homme est simultanément «pro-jeté » ${ }^{67}$ vers "l'avoir-été » ${ }^{68}$ de Béatrix (son portrait romanesque tel qu'il s'est inscrit en Calyste) et vers « l'à-venir » ${ }^{69} \mathrm{~d}^{\prime}$ une Béatrix qu'il ne connait pas encore : « Ce nom brillait à ses yeux comme un talisman, il y sentait je ne sais quoi de fatal ; il savait, sans en pouvoir douter, qu'il aimerait cette femme ; les plus petites choses qui la concernaient l'occupaient déjà, l'intéressaient et piquaient sa curiosité. ${ }^{70}$

62 D'un point de vue philosophique, «percevoir » implique d'organiser immédiatement ses sensations et de les interpréter, chose que Calyste ne semble pas capable de faire.

63 Balzac, Béatrix, p. 152.

64 Ibid.

65 Cf. Martin Heidegger, Etre et Temps [Sein und Zeit], trad. François Vezin, Paris, 1986, § 65.

66 Dans Être et Temps, Heidegger définit l'ekstase comme une temporalité « hors-de-soi originaire en et pour soi-même » («Außer-sich » an und für sich selbst, ibid., § 65).

67 En référence au Dasein, cf. ibid.

68 Ibid.

69 Ibid.

70 Balzac, Béatrix, p. 153. 
Or ce moment, qui pourrait être celui de la véritable rencontre de Calyste avec son objet d'admiration, correspond également à celui où l'on apprend que Calyste aime Béatrix : «Béatrix avait hérité de l'amour que dédaignait Camille ${ }^{71}$. Mais si l'amour se substitue si vite à l'admiration, qu'est devenue l'étape qui consiste à transformer l'objet d'admiration en objet de connaissance ? L'amour en ferait-il office ? Justement, la scène de la caisse se clôt sur une étrange réflexion du narrateur omniscient : «Un fait digne de remarque, et qui cependant n'a point été remarqué, c'est comme nous soumettons souvent nos sentiments à une volonté, combien nous prenons une sorte d'engagement avec nous-mêmes, et comme nous créons notre sort : le hasard n'y a certes pas autant de part que nous le croyons. $\gg^{72}$ Voilà qui suggère que Calyste, jusqu'à présent si peu disposé aux exercices de l'esprit, aurait fait naitre son amour pour Béatrix par une opération de l'esprit, puisque sa volonté aurait davantage de part dans ses sentiments pour elle que le «hasard». Mais cela suffit-il à démontrer que l'objet d'admiration est voué à se transformer en objet de connaissance par le biais de l'amour? Rien n'est moins sûr.

En effet, ce «fait digne de remarque », dont Balzac prétend qu'il « n'a point été remarqué », rappelle d'une étrange manière le concept de cristallisation proposé par Stendhal dans son essai De l'amour. La cristallisation suggère que dans l'amour, la volonté joue un rôle important. Or, pour Stendhal, l' «opération de l'esprit » qu'implique l'amour ne vise pas à connaître, mais tout au contraire à méconnaitre, ou plutôt à se mystifier soi-même : « Ce que j'appelle cristallisation, c'est l'opération de l'esprit qui tire de tout ce qui se présente la découverte que l'objet aimé a de nouvelles perfections. ${ }^{73}$ Ces perfections, l'objet aimé peut éventuellement s'en parer après-coup, mais elles ne sont pas là à l'origine. Stendhal utilise la métaphore du rameau « effeuillé par l'hiver » ${ }^{74}$ que l'on plonge dans le sel ${ }^{75}$ et que l'on ressort couvert « d'une infinité de diamants mobiles et éblouissants $»^{76}$, de telle sorte qu'« on ne peut plus reconnaître le

\footnotetext{
71 Ibid.

72 Ibid.

73 Cf. Stendhal, De l'amour, Paris, 2014, p. 64.

74 Ibid.

75 On peut difficilement s'empêcher de voir quelque clin d'œil à cette métaphore saline dans l'environnement choisi par Balzac pour cette scène de la malle : le dédale des marais salants et le navire qui transporte Béatrix, venu prendre sa cargaison de sel. Stendhal n'est d'ailleurs pas absent du roman, quoique jamais nommé. Il apparait sous les traits du premier amant de Félicité, un « célèbre inconnu », « un des esprits les plus originaux de [son] temps » qui « écrivait sous un pseudonyme » et dont les « premiers écrits annoncèrent un adorateur de l'Italie. » (Balzac, Béatrix, p. 105.)

76 Stendhal, De l'amour, p. 64.
} 
rameau primitif $\gg 77$. Ce processus de cristallisation dont, fait notable, l'admiration constitue pour Stendhal également la première étape, n'a donc pas pour conséquence la connaissance du vrai, mais la mystification.

Toute l'histoire de la relation de Calyste avec Béatrix en fait la démonstration. Calyste restera toujours fidèle à cet « engagement» pris avec lui-même devant le nom adoré. Il ne cessera jamais de compléter, d'enrichir, de prolonger le portrait romanesque de Béatrix. Le problème, c'est que Calyste n'est pas poète. Chez lui, la mystification n'a rien de commun avec le «mentir-vrai ${ }^{78}$ romanesque. Elle ne fait qu'entretenir sa difficulté à lire le monde et à se lire lui-même. Dans la dernière partie du roman ${ }^{79}{ }^{1} \mathrm{il} \mathrm{se}$ "perdra » auprès de Béatrix, comme on se perd dans un vice. Marié à Sabine de Granlieu, il retrouve Béatrix, dont il était sans nouvelles depuis des années, et devient son amant. Pour elle, et sur sa cruelle demande, il ment, dissimule et sème le malheur autour de lui. Il trahit toutes les valeurs bretonnes et catholiques dont il est pourtant l'incarnation. En d'autres termes, il ne se connait plus lui-même.

Quant à Béatrix, l'amour qui découle de l'admiration n'est pas non plus un gage de connaissance. Mais pour de tout autres raisons. Elle qui nous est initialement présentée comme une de ces femmes capables de tout abandonner, mari, enfant, position sociale, pour se donner entièrement à l'amour, semble curieusement très peu apte à aimer. Alain remarquait déjà cette impuissance de Béatrix dans sa relation avec Conti, pour qui elle a quitté son mari : «Le coup d'éclat de Béatrix partant avec Conti n'est que d'humeur. L'amour n'arrive pas à naître [...]. . ${ }^{80}$ D'après lui, la raison doit en être imputée au fait que chez elle, « [l] a vanité l'emporte, c'est-à-dire que l'attitude, l'apparence et même la toilette règlent les pensées ${ }^{81}$. C'est bien là qu'est le problème : Béatrix, qui maitrise l'artifice à la perfection veut à tout prix être l'objet d'admiration et ne peut véritablement porter son regard que sur elle-même. À tel point qu'elle peine à admirer d'autres objets. Chez elle, l'admiration commence toujours par une velléité, certes sincère, mais ne parvient jamais à s'enraciner. Camille nous l'avait déjà révélé dès le portrait qu'elle avait tracé à Calyste : «La marquise a

$77 \quad$ Ibid.

78 L'expression, empruntée à Louis Aragon, a déjà été utilisée pour qualifier certains procédés romanesques balzaciens, notamment celui employé par Diane de Maufrigneuse (cf. Balzac, Les Secrets de la princesse de Cadignan, Lausanne, 1968). Cf. Christelle Girard, «Canonisation du roman, pensée du romanesque : Balzac, Les Secrets de la princesse de Cadignan », in: Littérature, 2014, $\mathrm{n}^{\circ} 173, \mathrm{p} .35-53$.

79 Béatrix a fait l'objet de nombreuses éditions du vivant de Balzac. Initialement, il était divisé en plusieurs chapitres. En 1845, cette division a été supprimée. Un peu plus tard, le titre « Dernière partie » a été remplacé par «Un adultère rétrospectif» (Furne).

8 o Alain, «Avec Balzac», p. 92.

81 Ibid. 
eu pour moi la plus vive admiration, mais de l'admiration à la jalousie il n'y a qu'un pas. ${ }^{82}$ Béatrix jalouse ce qu'elle ne peut surpasser.

Ainsi, elle ne peut rien apprendre, rien connaitre de ce qui se trouve au-dessus d'elle. L'objet d'admiration ne s'inscrit jamais suffisamment longtemps comme tel dans son horizon perceptif pour se transformer en objet de connaissance. Chez elle, tout est extérieur, imitation. ${ }^{83}$ Elle appartient désespérément à ce Paris de la modernité postrévolutionnaire, où « tout fume, tout brûle, tout brille $[. ..]{ }^{84}$ etc., où rien ne peut se fixer, rien ne peut se connaitre. La romancière nous avait prévenus : Béatrix est un « ange », oui ; mais un « ange qui flambe et se dessèche ${ }^{85}$. Contrairement à Camille, Béatrix est incapable de percevoir l'objet qui se détache pourtant de l'horizon perceptif. Il faut qu'un être supérieur le lui montre, et là encore, le processus de l'admiration ne parvient pas à atteindre son terme.

Car au fond, ce que Béatrix admire en Calyste, c'est son admiration pour elle. Qu'est-ce que Calyste pour elle ? Un faire-valoir et un butin de guerre ; car se faire aimer de Calyste, c'est jouir enfin d'une supériorité sur l'inégalable Félicité : «Peut-être une des plus grandes jouissances que puissent éprouver les petits esprits ou les êtres inférieurs est-elle de jouer les grandes âmes et de les prendre à quelque piège. Béatrix savait être bien au-dessous de Camille Maupin. Cette infériorité n'existait pas seulement dans cet ensemble de choses morales appelé talent, mais encore dans les choses du cœur nommées passion. ${ }^{86}$ Quelle victoire sur une ennemie aussi redoutable !

Mais cette victoire, comme toutes les victoires de Béatrix, est une victoire de surface. Car l'admiration de Calyste, l'admiration au sens philosophique du terme, celle qui nous fait connaitre le monde sous un angle inattendu, c'est à Camille qu'elle revient. Depuis sa rencontre avec cette femme de génie (rencontre dont le récit ne nous dit rien), l'horizon perceptif de Calyste s'est considérablement élargi : «J'étais aussi loin de mon siècle que Guérande est loin de Paris. [Camille] a été un peu la mère de mon intelligence ${ }^{87}$, confiera Calyste à la baronne du Guénic.

Or quel est cet autre monde ? C'est la face apprésentée du monde pour Calyste. Mais ce n'est pas encore la face apprésentée du monde pour le lecteur. Cet autre monde - Paris, l'art, la culture, les belles œuvres, les voyages et les

\footnotetext{
82 Balzac, Béatrix, p. 125.

83 Elle essaie d'imiter le salon de Camille, mais n'est pas à la hauteur. Par ailleurs, Alain décrit Rubempré selon les mêmes termes (Alain, «Avec Balzac», p. 200).

84 Balzac, La Fille aux yeux d'or, p. 7 .

85 Balzac, Béatrix, p. 127.

86 Ibid., p. 227.

87 Ibid., p. 143 .
} 
langues étrangères - n'est pour le lecteur que l'autre face, parfaitement perceptible, visible, «présentée », du monde guérandais. Cela veut-il donc dire que l'objet d'admiration «Camille» n'est devenu objet de connaissance que pour Calyste?

Observons ceci : l'initiation de Calyste par Félicité consiste à décrypter les intentions et les intérêts dissimulés de la société, les hypocrisies, les double-jeux, les sentiments cachés, etc. En quelque sorte, elle lui apprend à lire le monde perceptible, ou plutôt les apparences du monde perceptible ; elle lui apprend à se frayer un chemin dans la complexité, l'hétérogène, bref, le monde «connu », celui qui n'excède pas les bornes de l'horizon perceptif. Ce monde qui semble évoluer, pour ainsi dire, suivant un axe horizontal, est un monde lisible pour le lecteur. Pour ce dernier, c'est donc ailleurs que se joue véritablement la transformation de l'objet d'admiration en objet de connaissance.

Et c'est Félicité qui nous ouvre la voie de cette transformation. Cela peut paraître étrange, dans la mesure où elle semble nous dissuader de l'existence d'une face apprésentée du monde, plutôt qu'elle ne nous la suggère : elle devine tout, anticipe tout, sait tout. Rien, sur cet axe horizontal du monde, n'échappe à sa sagacité. Pour elle, le monde est comme dépourvu de mystère. Or c'est elle qui nous suggère l'existence d'un point aveugle dans notre horizon perceptif. Et si ce point a échappé à notre perception, c'est parce qu'il ne se situe pas sur l'axe horizontal du monde balzacien.

Comprenons bien ceci : Félicité est fatiguée du monde horizontal, elle en a fait le tour. Si Calyste, cette beauté au cœur pur, a fait saillie sur son horizon perceptif, c'est à la manière d'un signe, d'un rappel à un au-delà de l'horizon perceptif, d'un rappel à l'infini. En s'abîmant en lui, suivant un axe vertical, Félicité se départit des limites de l'horizontalité, qui l'épuisent : «Mon âme se repose dans son cœur de toutes les corruptions, de toutes les idées de la science, de la littérature, du monde, de la politique, de tous ces inutiles accessoires sous lesquels nous étouffons le bonheur. » Après Calyste, il n'y a pas d'autre salut que Dieu.

Là où Béatrix n'a su voir qu'un faire-valoir, Camille a vu un chef-d'œuvre de la nature, qui est plus que la nature :

Ah ! ma chère, quand vous combattrez l'âme angélique d'un pareil enfant, vous me comprendrez. Chez lui, la beauté n'est rien, il faut pénétrer dans ce cœur pur, dans cette naïveté surprise à chaque pas fait dans le royaume de l'amour. Quelle foi ! quelle candeur ! quelle grâce ! Les anciens avaient raison dans le culte qu'ils rendaient à la sainte beauté. [...] La beauté, ma chère, est le génie des choses ; elle est l'enseigne que la nature a mise à ses créations les plus parfaites, elle est le plus vrai des symboles, comme elle est le plus grand des hasards. [...] Qui nous 
a fait rester des heures entières devant certains tableaux en Italie, où le génie a cherché pendant des années à réaliser un de ces hasards de la nature ? $^{88}$

Derrière la beauté de Calyste, il y a à la fois «le plus vrai des symboles » et «le plus grand des hasards». En d'autres termes, derrière Calyste, il y a le mystère de la création. Calyste est «symbole» parce qu'il est promesse de sens, «hasard», parce qu'il ne peut être expliqué. Ce mystère de la création est certes double : il concerne aussi bien l'artiste que Dieu. Or, c'est sur cette ambivalence de la création que va se jouer la métamorphose de l'objet d'admiration en objet de connaissance : Félicité ne sera apte à se pencher sur le mystère divin - c'est-à-dire à se retirer au couvent - qu'une fois elle aura épuisé toutes les possibilités de son art, qu'une fois qu'elle aura épuisé Camille Maupin, jusqu'à la tuer en elle. Calyste sera l'objet de son dernier roman. Ce roman n'est pas de ceux qu'elle écrit, mais de ceux qu'elle «fait», et que Béatrix redoute ${ }^{89}$.

Comprenant que Calyste est perdu pour elle, la romancière se fait démiurge et se lance dans la création in vivo du bonheur de Calyste. Il désire que Béatrix l'aime ? Elle crée l'histoire de Béatrix répondant à ses hommages. Mais, toujours devancée par son propre génie, elle compose ce roman en sachant que la réponse de la marquise ne signifie rien, qu'elle est vide, comme Béatrix ; et comme elle, toute d'apparence. Félicité sait que la vaine marquise ne peut pas aimer Calyste. Béatrix quitte les Touches pour suivre Conti en Italie, prise de court : elle n'a pas songé à former le moindre projet pour Calyste. Fin de la première partie du roman de Camille. Il faut songer à consoler Calyste de son chagrin. Deuxième partie du roman de Camille. Elle lui compose une vie en accord avec sa nature bretonne. Elle choisit pour lui Sabine de Granlieu, un personnage qui présente quelque ressemblance physique avec Béatrix. Elle orchestre son mariage, lui lègue sa fortune. Fin du roman de Camille Maupin, fin du « pauvre Camille, qui n'est plus $»^{90}$. D'ailleurs, le roman Béatrix s'arrêtait initialement là. Ce n'est que plus tard ${ }^{91}$ que Balzac a décidé de le prolonger par ce qui devait être un autre roman : un roman qui, bien qu'il concerne Calyste et Béatrix, est en effet une autre histoire. ${ }^{92}$

88 Ibid., p. 206-207.

89 Ibid., p. 234: « Les romans que vous faites, ma chère, sont un peu plus dangereux que ceux que vous écrivez, dit la marquise. »

$90 \quad$ Ibid., p. 286.

$91 \quad$ En 1844.

92 Alain dira de cette dernière partie qu'il «veu[t] la négliger» parce qu'elle «semble un autre roman, parisien celui-là, où les passions s'usent, se dégradent, et finalement se perdent dans un tissu d'intrigues et d'intérêts, et par des causes diverses et convergentes » 
Phénomène étonnant, Camille Maupin se compte elle-même au nombre des personnages : elle organise, met en scène sa sortie du roman par le biais d'une lettre qui est remise à Calyste au moment précis de la signature des contrats de mariage et dans laquelle elle répond, comme par anticipation, à tous les doutes qui assaillent le futur marié : «Calyste, avant d'entrer dans ma cellule de novice, il m'est permis de jeter un regard sur le monde que je vais quitter pour m'élancer dans le monde de la prière. Ce regard est entièrement à vous, qui, dans ces derniers temps, avez été pour moi tout le monde. Ma voix arrivera, si mes calculs ne m'ont point trompée, au milieu d'une cérémonie à laquelle il m'était impossible d'assister. ${ }^{93}$

Cette lettre, à caractère didactique est un manuel de lecture du monde; ${ }^{94}$ et de soi. Mais elle est également le testament de l'écrivain qui n'écrira plus, du personnage qui sort du roman. Camille Maupin, en se composant elle-même dans le drame créé pour Calyste, est arrivée au bout de ses propres possibilités et peut enfin devenir Félicité.

Ce nom, au début du roman, elle le trouvait pourtant mal choisi : «Sterne a raison : les noms signifient quelque chose, et le mien est la plus sauvage raillerie. Je mourrai sans trouver chez un homme l'amour que j'ai dans le cœur, la poésie que j'ai dans l'âme. ${ }^{95}$ La Félicité du début du roman n'avait cependant pas tout à fait tort : rien d'assez grand, rien d'assez beau n'a pu, dans le monde des hommes, soutenir son exceptionnalité. Calyste, son objet d'admiration, fut avant tout objet de poésie, comme l'était sans doute Béatrix pour Dante. De la rencontre entre cet objet et le poète est née l'œuvre poétique, et de l'œuvre poétique accomplie est né la transcendance. La Béatrix de Balzac ne pouvait être, pour Calyste, ce premier pas vers un au-delà de l'horizon perceptif. En elle-même, Béatrix n'est qu'une coquille vide, une forme, certes belle, mais sans contenu ; un signe qui ne signifie rien. Elle ne se charge de sens que par l'admiration qu'on lui voue, et ce sens diffère selon qui l'admire. Béatrix est le piège de l'amant et le trésor du poète.

Dans sa dernière lettre, Camille se félicite d'avoir accompli son devoir en assurant le bonheur de Calyste. Elle a surtout accompli son devoir de poète. Ce grand personnage peut dès lors quitter le monde, ou du moins l'axe horizontal du monde : «- Après l'homme, il n'y a plus que Dieu, répondit gravement la femme célèbre [à Béatrix]. Dieu, c'est l'inconnu. Je m’y jetterai comme

(Alain, «Avec Balzac», p. 90). Nous ne l'aborderons pas non plus : l'admiration y tient trop peu de place.

93 Balzac, Béatrix, p. 285.

94 On retrouve là la veine des lettres envoyées à Félix par Henriette de Mortsauf dans Le Lys dans la vallée.

Balzac, Béatrix, p. 119. 
dans un abîme. ${ }^{96}$ Après une telle élévation, persister à rester au milieu des hommes, c'est se condamner, comme le grand peintre Frenhofer, ${ }^{97}$ à y être à jamais incompris, illisible. Le roman atteint là les limites de la représentation. Tout ce qui se situe au-delà de l'horizon perceptif est de l'ordre de l'indicible, de l'invisible, du non-communicable et ne peut plus guère quêtre suggéré.

Laccès que confère l'objet d'admiration à une connaissance verticale du monde est réservé à Félicité. Pour le lecteur, elle ne fait que s'esquisser au large de son horizon perceptif. Mais elle se précise, à mesure du roman, jusquà la dernière limite de la représentation. D'objet d'admiration en objet d'admiration, nous avons été soumis à un véritable parcours initiatique. Nous avons d'abord admiré la Bretagne, par opposition à Paris, puis Paris, par opposition à la Bretagne ; et Balzac nous a fait connaitre qu'il s'agissait d'un seul et même monde. Nous nous attendions, dès le titre, à admirer Béatrix, nous avons finalement admiré Félicité. Le récit a fait se démarquer Calyste de l'horizon perceptif, mais nous sommes revenus à Félicité, que nous avons connue toujours plus grande, toujours plus admirable, inaccessible, ainsi que le récit balzacien l'a construite. Puis nous avons vu naitre l'admiration de Calyste pour Béatrix et celle de Béatrix pour Calyste. C'était encore admirer Camille Maupin, romancière démiurge, dans la réalisation de son ultime chef-d'œuvre. Nous l'avons contemplée en train de démêler ces signes inextricables que sont les objets d'admiration, tandis que le récit balzacien déroulait pour nous le mystérieux objet d'admiration « Félicité ».

Dans son essai « Avec Balzac», Alain se délectait d'intituler son étude de Béatrix « Félicité » ${ }^{98}$ : il y trouvait « une sorte de justice » ${ }^{99}$. Il eût été juste, en effet, que le roman porte le nom de celle qui en est le véritable centre. Juste, mais peu stratégique : nous devions nous laisser mystifier par Balzac, nous devions nous laisser surprendre par Félicité, cet exceptionnel objet d'admiration, par qui nous avons pu entrevoir la dernière extrémité de la face apprésentée du monde et connaitre cet «auguste mensonge ${ }^{100}$ qu'est le roman balzacien.

\footnotetext{
$96 \quad$ Ibid., p. 201.

97 Frenhofer atteint une telle perfection dans son art qu'il dépasse les capacités de la représentation : son chef-d'œuvre n'est qu'un tas de « couleurs confusément amassées », « une espèce de brouillard informe » (Balzac, Le Chef-d'œuvre inconnu, p. 55). Sa toile est illisible.

98 Cf. Alain, «Avec Balzac», p. 89.

99 Ibid.

100 Cf. Honoré de Balzac, Avant-propos à La Comédie humaine, Paris, 1855, p. 26.
} 